\title{
Author Correction: Mapping local patterns of childhood overweight and wasting in low- and middle-income countries between 2000 and 2017
}

\author{
LBD Double Burden of Malnutrition Collaborators \\ Correction to: Nature Medicine https://doi.org/10.1038/s41591-020-0807-6, published online 20 April 2020.
}

In the version of this article initially published, an author's name was incorrect (Yesdhambel T. Nigatu). The correct name is 'Yeshambel T. Nigatu'. Another author's name and affiliation were incorrect (Mowafa Housseh; Division of Information and Computing Technology, Hamad Bin Khalifa University, Doha, Qatar). The correct name and affiliation are 'Mowafa Househ' and 'College of Science and Engineering, Hamad Bin Khalifa University, Doha, Qatar'. Also, author Ewerton Cousin (affiliation, Postgraduate Program in Epidemiology, Federal University of Rio Grande do Sul, Porto Alegre, Brazil) was not listed. This author has now been included. The errors have been corrected in the HTML and PDF versions of the article.

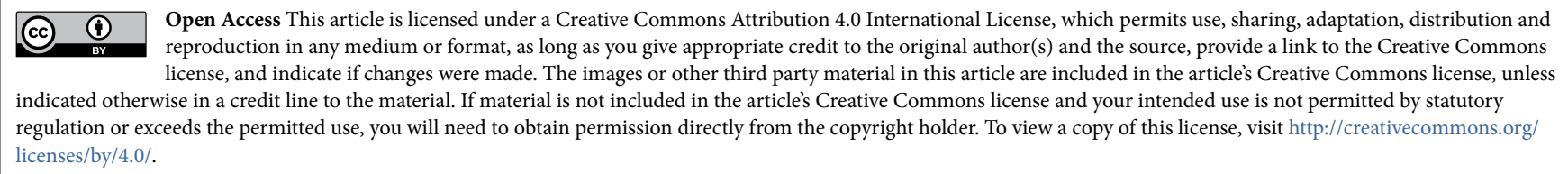

Published online: 2 July 2020

https://doi.org/10.1038/s41591-020-0972-7

(๑) The Author(s) 2020

\section{Author Correction: Glomerular filtration rate by differing measures, albuminuria and prediction of cardiovascular disease, mortality and end-stage kidney disease}

\author{
Jennifer S. Lees (D), Claire E. Welsh, Carlos A. Celis-Morales, Daniel Mackay, James Lewsey, Stuart R. Gray (D), \\ Donald M. Lyall, John G. Cleland (D), Jason M. R. Gill, Pardeep S. Jhund, Jill Pell, Naveed Sattar (D), Paul Welsh and \\ Patrick B. Mark (iD
}

Correction to: Nature Medicine https://doi.org/10.1038/s41591-019-0627-8, published online 7 November 2019.

In the version of this article initially published, there were errors in the categorization of uACR into groups of $<3,3-30$ and $>30 \mathrm{mg} \mathrm{mmol}^{-1}$ : an incorrect proportion of participants were categorized into the $>30 \mathrm{mg} \mathrm{mmol}^{-1}$ group, but analyses that used uACR as a continuous variable are unaffected. There was an additional error in the calculation of eGFRcr-cys for a small subgroup of women. Consequently, in the 'Demographics of participants' section in the Results, the $r$ values between eGFRcys and eGFRcr-cys and between eGFRcr and eGFRcr-cys have been updated; in the 'Prediction of all-cause mortality and CVD with albuminuria' section in the Results, "the risk of ESKD increases with higher uACR category" has been changed to "the risk of all outcomes generally increases with higher uACR category"; in the Results, the values in Tables 1-4 have been updated; Figs. 2 and 3 and Supplementary Tables 1-9 have been revised to include updated values, and the Fig. 2 legend has also been updated accordingly; in the Discussion, "In the UK Biobank, only $3.2 \%$ of 440,526 participants had uACR $>30 \mathrm{mg} \mathrm{mmol}^{-1}$ " has been changed to "In the UK Biobank, only $0.4 \%$ of 440,526 participants had uACR > $30 \mathrm{mg} \mathrm{mmol}^{-1}$ "; and in the Methods, "categorical NRI was tested for reclassification of patients from 'low' to 'intermediate' risk" has been changed to "categorical NRI was tested for reclassification of patients from 'low' to 'high' risk." The main messages of the paper are unaffected. The errors have been corrected in the HTML and PDF versions of the article.

Published online: 15 July 2020

https://doi.org/10.1038/s41591-020-0996-Z

(C) The Author(s), under exclusive licence to Springer Nature America, Inc. 2020 